\title{
Subring dan Ideal pada Ring JR-2CN dan JR-3CN
}

\author{
${ }^{1}$ Julana S. Rarung, ${ }^{2}$ Mans L. Mananohas, ${ }^{3}$ Luther Latumakulita \\ ${ }^{1}$ Program Studi Matematika, FMIPA, UNSRAT, julanastefani@gmail.com \\ ${ }^{2}$ Program Studi Matematika, FMIPA, UNSRAT, mansmananohas@yahoo.com \\ ${ }^{3}$ Program Studi Matematika, FMIPA, UNSRAT, latumakulitala@ unsrat.ac.id
}

\begin{abstract}
Abstrak
Ring adalah himpunan dengan dua operasi biner dan memenuhi semua aksioma Ring. Adapun dua himpunan yang telah dibuktikan bahwa keduanya merupakan Ring yaitu, himpunan pasangan terurut dari bilangan bulat baru JR - 2CN dan JR - 2CN. Dalam tulisan ini, akan ditunjukkan beberapa Subring maupun Ideal pada Ring JR - 2CN dan JR - 2CN.
\end{abstract}

Kata kunci : Ideal, JR - 2CN, JR - 2CN, Subring

\section{Subring and Ideal Of Ring JR-2CN and JR-3CN}

\begin{abstract}
Ring is set with two binary operations and satisfy all of axioms Ring. There are two sets that was proven that both of them are Ring that are sets ordered pairs of new integer, JR-2CN and JR-3CN. In this research, will be shown some Subring and Ideal of Ring JR $-2 C N$ and JR $-3 C N$.
\end{abstract}

Key words : Ideal, JR - 2CN , JR - 3CN, Subring

\section{Pendahuluan}

Ring adalah himpunan dengan dua operasi biner dan memenuhi semua aksioma Ring. Salah satu contoh himpunan yang merupakan Ring adalah Himpunan bilangan Bulat. Pada tahun 2010, Rand Alfaris dan Hailiza Kamarulhaili menemukan himpunan dua pasangan terurut dari bilangan bulat baru JR $-2 \mathrm{CN}$ dan JR $-3 \mathrm{CN}$. JR $-2 \mathrm{CN}$ merupakan himpunan bilangan bulat yang dapat ditulis dalam bentuk penjumlahan 2 bilangan bulat berpangkat 3. Sedangkan, JR - 3CN merupakan himpunan bilangan bulat yang dapat ditulis dalam bentuk perjumlahan 3 bilangan bulat berpangkat 3. Dua himpunan bilangan bulat $J R-2 C N$ dan JR - 3CN merupakan grup Abelian serta keduanya isomorfik [1]. Grup abelian baru ini rencananya akan diterapkan dalam Kriptografi khususnya dalam keamanan kunci publik. Hasil riset sebelumnya membuktikan bahwa JR - 2CN dan JR - 3CN adalah Ring dan Lapangan [2].

Berdasarkan penelusuran pustaka, kerja riset yang masih perlu dilakukan berkaitan dengan tipe Ring ini adalah mengkaji Subring dan Ideal dari Ring JR - 2CN dan JR - 3CN.

\section{Subring dan Ideal}

Definisi 1 (Subring)

Sebuah subset $S$ pada suatu Ring $R$, disebut Subring pada $R$ jika $S$ juga memenuhi semua aksioma Ring [3].

\section{Teorema 1}

Sebuah subset $S$ pada suatu Ring $R$ disebut Subring pada $R$ jika memenuhi [3] :

1. $0 \in S$,

2. Untuk setiap $x, y \in S$, maka $x-y \in S$,

3. Untuk setiap $x, y \in S$, maka $x y \in S$

Definisi 2 (Ideal)

Suatu Subring $\mathrm{N}$ dari Ring $\mathrm{R}$ yang bersifat $a \mathrm{~N} \subseteq \mathrm{N}$ dan $\mathrm{Nb} \subseteq \mathrm{N}, \forall a, b \in R$ disebut Ideal [4]. 


\section{JR $-2 \mathrm{CN}$}

Setiap anggota dari $J R-2 C N$ adalah dua pasangan terurut yang komponennya adalah dua bilangan bulat $(j, r)[4]$.

$J R-2 C N=\left\{(j, r) \in Z \mathrm{XZ}: j^{3}+r^{3}=6 \alpha x^{2}+2 \alpha^{3}\right.$ dimana $\left.j=x+\alpha, r=\alpha-x \quad \forall \alpha, x \in Z\right\}$

\section{Definisi 3}

$\forall\left(j_{1}, r_{1}\right),\left(j_{2}, r_{2}\right) \in J R-2 C N$ dimana, $j_{i}=x_{i}+\alpha_{i}$ dan $r_{i}=\alpha_{i}-x_{i}$ untuk $i=1,2$ maka terhadap operasi $\bigoplus_{J R-2 C N}$ berlaku [1] :

$$
\left(j_{1}, r_{1}\right) \oplus_{J R-2 C N}\left(j_{2}, r_{2}\right)=\left(j_{1}+j_{2}, r_{1}+r_{2}\right)
$$

Jika dan hanya jika,

$$
\left(j_{1}+j_{2}\right)^{3}+\left(r_{1}+r_{2}\right)^{3}=6\left(\alpha_{1}+\alpha_{2}\right)\left(x_{1}+x_{2}\right)^{2}+2\left(\alpha_{1}+\alpha_{2}\right)^{3}
$$

JR - 2CN memiliki elemen nol $(0,0)$ untuk setiap elemen $(j, r)$

\section{Definisi 4}

Untuk setiap $\left(j_{1}, r_{1}\right),\left(j_{2}, r_{2}\right) \in J R-2 C N$ dimana, $j_{i}=x_{i}+\alpha_{i}$ dan $r_{i}=\alpha_{i}-x_{i}, \forall i=1,2$.

Berlaku [2] :

$$
\left(j_{1}, r_{1}\right) \odot_{J R-2 C N}\left(j_{2}, r_{2}\right)=\left(\frac{j_{1} \cdot j_{2}+r_{1} \cdot r_{2}}{2}, \frac{j_{1} \cdot r_{2}+j_{2} \cdot r_{1}}{2}\right)
$$

Jika dan hanya jika,

$$
\left(j_{1}+j_{2}\right)^{3}+\left(r_{1}+r_{2}\right)^{3}=6\left(\alpha_{1} \cdot \alpha_{2}\right)\left(x_{1} \cdot x_{2}\right)^{2}+2\left(\alpha_{1} \cdot \alpha_{2}\right)^{3}
$$

JR - 2CN memiliki elemen satuan $(2,0)$ untuk setiap elemen $(j, r)$.

\section{JR $-3 \mathrm{CN}$}

Setiap anggota dari grup $J R-3 C N$ terdiri dari pasangan terurut $(j, r)_{\alpha}$, yang didefinisikan sebagai berikut [4] :

$J R-3 C N=\left\{(j, r) \in Z X Z: j^{3}+r^{3}-\alpha^{3}=3 \alpha r^{2}-3 \alpha^{2} r\right.$, dimana $\left.j=\alpha-r \quad \forall \alpha, r \in Z\right\}$.

\section{Definisi 5}

$\forall\left(j_{1}, r_{1}\right)_{\alpha_{1}},\left(j_{2}, r_{2}\right)_{\alpha_{2}} \in \mathrm{JR}-3 \mathrm{CN}$ dimana, $j_{i}=\alpha_{i}-r_{i}$, untuk $i=1,2 . \bigoplus_{J R-3 C N}$ definisikan pada $J R-3 C N$ sedemikian sehingga [1],

$$
\left(j_{1}, r_{1}\right)_{\alpha_{1}} \bigoplus_{J R-3 C N}\left(j_{2}, r_{2}\right)_{\alpha_{2}}=\left(j_{1}+j_{2}, r_{1}+r_{2}\right)_{\alpha_{1}+\alpha_{2}}
$$

Jika dan hanya jika,

$$
\left(j_{1}, j_{2}\right)^{3}+\left(r_{1}+r_{2}\right)^{3}-\left(\alpha_{1}+\alpha_{2}\right)^{3}=3\left(\alpha_{1}+\alpha_{2}\right)\left(r_{1}+r_{2}\right)^{2}-3\left(\alpha_{1}+\alpha_{2}\right)^{2}\left(r_{1}+r_{2}\right) .
$$

JR $-3 \mathrm{CN}$ memiliki elemen nol $(0,0)_{0}$ untuk setiap elemen $(j, r)_{\alpha}$.

\section{Definisi 6}

Untuk setiap, $\left(j_{1}, r_{1}\right)_{\alpha_{1}}{ }^{\prime}\left(j_{2}, r_{2}\right)_{\alpha_{2}} \in \mathrm{JR}-3 \mathrm{CN}$ dimana, $j_{i}=\alpha_{i}-r_{i}$ untuk $i=1,2$ terhadap operasi biner $\odot_{J R-3 C N}$ berlaku [2],

$$
\left(j_{1}, r_{1}\right)_{\alpha_{1}} \odot_{J R-3 C N}\left(j_{2}, r_{2}\right)_{\alpha_{2}}=\left(j_{1} \cdot j_{2}+j_{1} \cdot r_{2}+j_{2} \cdot r_{1}, r_{1} \cdot r_{2}\right)_{\alpha 1 \cdot \alpha 2}
$$

Jika dan hanya jika,

$$
\left(j_{1}, j_{2}\right)^{3}+\left(r_{1}+r_{2}\right)^{3}-\left(\alpha_{1}+\alpha_{2}\right)^{3}=3\left(\alpha_{1} \cdot \alpha_{2}\right)\left(r_{1} \cdot r_{2}\right)^{2}-3\left(\alpha_{1} \cdot \alpha_{2}\right)^{2}\left(r_{1} \cdot r_{2}\right)
$$

JR $-3 \mathrm{CN}$ memiliki elemen satuan $(0,1)_{1}$ untuk setiap elemen $(j, r)_{\alpha}$. 


\section{Metode Penelitian}

Penelitian ini dilakukan berdasarkan studi literatur berupa buku-buku dan jurnal-jurnal ilmiah mengenai Ring JR $-2 \mathrm{CN}$ dan JR $-3 \mathrm{CN}$.

\section{Hasil dan Pembahasan}

6.1 Beberapa contoh Subring dari $\left(\mathrm{JR}-2 \mathrm{CN}, \bigoplus_{\mathrm{JR}-2 \mathrm{CN}}, \odot_{\mathrm{JR}-2 \mathrm{CN}}\right)$

dan $\left(\mathbf{J R}-\mathbf{3 C N}, \oplus_{\mathbf{J R}-3 \mathbf{C N}}, \odot_{\mathrm{JR}-3 \mathrm{CN}}\right)$

Contoh Subring pada JR - 2CN yaitu :

\section{Preposisi 1}

$\left(K, \bigoplus_{J R-2 C N}, \odot_{J R-2 C N}\right)$ dimana $K=\{(j, j) \in J R-2 C N, \forall j \in \mathbb{Z}\}$

Bukti

1. Misalkan $(m, m) \in K$ sembarang, disini jelas ada elemen nol $(0,0) \in K$. sehingga,

$$
(m, m) \bigoplus_{J R-2 C N}(0,0)=(m+0, m+0)=(m, m) .
$$

2. Akan ditunjukkan, $(m, m) \bigoplus_{J R-2 C N}(-n,-n)=(m-n, m-n) \in K$ $\forall(m, m),(n, n) \in K$ dimana,

$$
\begin{aligned}
& m=x_{1}+\alpha_{1} \\
& m=\alpha_{1}-x_{1}
\end{aligned}
$$

Dengan mensubtitusi persamaan (1) dan (2), diperoleh $m=\alpha_{1}$ dan $x_{1}=0$ untuk setiap $\alpha_{1} \in \mathbb{Z}$. Sedangkan untuk $(n, n)$ yaitu,

$$
\begin{aligned}
& n=x_{2}+\alpha_{2} \\
& n=\alpha_{2}-x_{2}
\end{aligned}
$$

Dengan cara yang sama diperoleh $n=\alpha_{2}$ dan $x_{2}=0$, untuk setiap $\alpha_{2} \in \mathbb{Z}$. Akibatnya berlaku,

$$
\begin{aligned}
(m-n)^{3}+(m-n)^{3} & =\left(\alpha_{1}-\alpha_{2}\right)^{3}+\left(\alpha_{1}-\alpha_{2}\right)^{3} \\
& =2\left(\alpha_{1}-\alpha_{2}\right)^{3} \\
& =0+2\left(\alpha_{1}-\alpha_{2}\right)^{3} \\
& =6\left(\alpha_{1}-\alpha_{2}\right) \cdot 0^{2}+2\left(\alpha_{1}-\alpha_{2}\right)^{3} \\
& =6\left(\alpha_{1}-\alpha_{2}\right)\left(x_{1}-x_{2}\right)^{2}+2\left(\alpha_{1}-\alpha_{2}\right)^{3}
\end{aligned}
$$

Perhatikan, disini jelas terdapat $x=x_{1}-x_{2}=0$ dan $\alpha=\alpha_{1}-\alpha_{2} \in \mathbb{Z}$, sehingga dapat diklaim $(m, m) \bigoplus_{J R-2 C N}(-n,-n)=(m-n, m-n) \in K$.

3. Akan ditunjukkan $(m, m) \odot_{J R-2 C N}(n, n) \in K, \forall(m, m),(n, n) \in K$. dari definisi 4 diperoleh,

$$
\begin{aligned}
(m, m) \odot_{J R-2 C N}(n, n) & =\left(\frac{m \cdot n+m \cdot n}{2}, \frac{m \cdot n+m \cdot n}{2}\right) \\
& =\left(\frac{2(m \cdot n)}{2}, \frac{2(m \cdot n)}{2}\right) \\
& =(m \cdot n, m \cdot n)
\end{aligned}
$$

Dengan mensubtitusi persamaan (1) dan (2), dan persamaan (3) dan (4). Akibatnya,

$$
\begin{aligned}
(m \cdot n)^{3}+(m \cdot n)^{3} & =\left(\alpha_{1} \cdot \alpha_{2}\right)^{3}+\left(\alpha_{1} \cdot \alpha_{2}\right)^{3} \\
& =2\left(\alpha_{1} \cdot \alpha_{2}\right)^{3} \\
& =0+2\left(\alpha_{1} \cdot \alpha_{2}\right)^{3} \\
& =6\left(\alpha_{1} \cdot \alpha_{2}\right) \cdot 0^{2}+2\left(\alpha_{1} \cdot \alpha_{2}\right)^{3} \\
& =6\left(\alpha_{1} \cdot \alpha_{2}\right) \cdot\left(x_{1} \cdot x_{2}\right)^{2}+2\left(\alpha_{1} \cdot \alpha_{2}\right)^{3}
\end{aligned}
$$

Karena, ada $x=x_{1} \cdot x_{2}=0 \quad \in \mathbb{Z}$ dan $\alpha=\alpha_{1} \cdot \alpha_{2} \in \mathbb{Z}$, maka jelas :

$$
(m, m) \odot_{J R-2 C N}(n, n)=(m n, m n) \in K .
$$




\section{Preposisi 2}

$\left(M, \oplus_{\mathrm{JR}-2 \mathrm{CN}}, \odot_{\mathrm{JR}-2 \mathrm{CN}}\right)$ dimana $M=\{(j,-j) \in \mathrm{JR}-2 \mathrm{CN}, \forall j \in \mathbb{Z}\}$

Bukti.

akan ditunjukkan $M$ merupakan Subring pada JR - 2CN. Perhatikan bahwa:

1. Misalkan, $\left(j_{1},-j_{1}\right) \in M$ sembarang maka ada elemen nol $(0,0) \in M$ sehingga berlaku,

$$
\left(j_{1},-j_{1}\right) \bigoplus_{J R-2 C N}(0,0)=\left(j_{1},-j_{1}\right)
$$

2. Akan ditunjukkan berlaku untuk setiap pernyataan berikut:

$$
\forall\left(j_{1},-j_{1}\right),\left(j_{2},-j_{2}\right) \in M \Rightarrow\left(\left(j_{1},-j_{1}\right) \oplus_{\mathrm{JR}-2 \mathrm{CN}}\left(-j_{2},-\left(-j_{2}\right)\right) \in M .\right.
$$

Dari definisi 3 diperoleh:

$$
\left(j_{1},-j_{1}\right) \oplus_{\mathrm{JR}-2 \mathrm{CN}}\left(-j_{2},-\left(-j_{2}\right)\right)=\left(j_{1}-j_{2},-\left(j_{1}-j_{2}\right)\right)
$$

Selanjutnya, misalkan, $j_{1}=x_{1}+\alpha_{1}$ dan $-j_{1}=\alpha_{1}-x_{1}$. Dengan mensubtitusi kedua persamaan tersebut diperoleh $j_{1}=x_{1}$ dan $\alpha_{1}=0$. Misalkan juga $j_{2}=x_{2}+\alpha_{2}$ dan $-j_{2}=\alpha_{2}-x_{2}$. Dengan cara yang sama, diperoleh $j_{2}=x_{2}$ dan $\alpha_{2}=0$. sehingga, berlaku:

$$
\begin{aligned}
\left(j_{1}-j_{2}\right)^{3}+\left(-\left(j_{1}-j_{2}\right)\right)^{3} & =6\left(\alpha_{1}-\alpha_{2}\right)\left(x_{1}-x_{2}\right)^{2}-2\left(\alpha_{1}-\alpha_{2}\right)^{3} \\
& =6(0) \cdot\left(x_{1}-x_{2}\right)^{2}-2(0)^{3}
\end{aligned}
$$

Karena, terdapat $\alpha=0 \in \mathbb{Z}$ dan $x=x_{1}-x_{2} \in \mathbb{Z}$. sehingga jelas,

$$
\left(\left(j_{1},-j_{1}\right) \bigoplus_{J R-2 C N}\left(-j_{2},-\left(-j_{2}\right)\right)=\left(j_{1}-j_{2},-\left(j_{1}-j_{2}\right)\right) \in M .\right.
$$

3. untuk setiap $\left(j_{1},-j_{1}\right),\left(j_{2},-j_{2}\right) \in M$ akan ditunjukkan, $\left(j_{1},-j_{1}\right) \odot_{J R-2 C N}\left(j_{2},-j_{2}\right) \in M$. Dengan menggunakan Definisi $4\left(\odot_{J R-2 C N}\right)$ diperoleh,

Perhatikan bahwa,

$$
\begin{aligned}
\left(j_{1},-j_{1}\right) \bigodot_{J R-2 C N}\left(j_{2},-j_{2}\right) & =\left(\frac{j_{1} \cdot j_{2}+\left(-j_{1}\right) \cdot\left(-j_{2}\right)}{2}, \frac{j_{1} \cdot\left(-j_{2}\right)+j_{2} \cdot\left(-j_{1}\right)}{2}\right) \\
& =\left(j_{1} \cdot j_{2},-\left(j_{1} \cdot j_{2}\right)\right)
\end{aligned}
$$

$$
\begin{aligned}
\left(j_{1} \cdot j_{2}\right)^{3}+\left(-\left(j_{1} \cdot j_{2}\right)^{3}\right. & =6\left(\alpha_{1} \cdot \alpha_{2}\right)\left(x_{1} \cdot x_{2}\right)^{2}-\left(\alpha_{1} \cdot \alpha_{2}\right)^{3} \\
& =6(0) \cdot\left(x_{1} \cdot x_{2}\right)^{2}-(0)^{3}
\end{aligned}
$$

Karena ada $\alpha=0 \in \mathbb{Z}, x=x_{1} \cdot x_{2} \in \mathbb{Z}$. sehingga dapat diklaim bahwa:

$$
\left(j_{1},-j_{1}\right) \bigodot_{\mathrm{JR}-2 \mathrm{CN}}\left(j_{2},-j_{2}\right)=\left(j_{1} \cdot j_{2},-\left(j_{1} \cdot j_{2}\right)\right) \in M .
$$

Contoh Subring pada JR - 3CN yaitu,

\section{Preposisi 3}

$\left(\mathrm{S}, \oplus_{J R-3 C N}, \odot_{J R-3 C N}\right)$ dimana $S=\left\{(-r, r)_{0} \in \mathrm{JR}-3 \mathrm{CN}: \forall r \in \mathbb{Z}\right\}$

Bukti.

akan dibuktikan S adalah Subring dari JR - 3CN. Perhatikan,

1. Misalkan, $(-r, r)_{0} \in S$, maka ada elemen $(0,0)_{0} \in S$, sehingga berlaku:

$$
\begin{aligned}
(-r, r)_{0} \bigoplus_{J R-3 C N}(0,0)_{0} & =(-r+0, r+0)_{0} \\
& =(-r, r)_{0}
\end{aligned}
$$

2. Akan ditunjukkan $\forall\left(-r_{1}, r_{1}\right)_{0},\left(-r_{2}, r_{2}\right)_{0} \in S$, berlaku :

$$
\left(-r_{1}, r_{1}\right)_{0} \oplus_{J R-3 C N}\left(-\left(-r_{2}, r_{2}\right)_{0}\right) \in S
$$


Perhatikan bahwa:

$$
\begin{aligned}
\left(-r_{1}, r_{1}\right)_{0} \bigoplus_{J R-3 C N}\left(r_{2},-r_{2}\right)_{0} & =\left(-r_{1}+r_{2}, r_{1}-r_{2}\right)_{0} \\
& =\left(-\left(r_{1}-r_{2}\right), r_{1}-r_{2}\right)_{0}
\end{aligned}
$$

Karena, $-\left(r_{1}-r_{2}\right) \in \mathbb{Z}$, dan $r_{1}-r_{2} \in \mathbb{Z}$, maka dapat disimpulkan:

$$
\left(-r_{1}, r_{1}\right)_{0} \oplus_{J R-3 C N}\left(-r_{2}, r_{2}\right)_{0} \in S .
$$

3. akan ditunjukkan $\forall\left(-r_{1}, r_{1}\right)_{0},\left(-r_{2}, r_{2}\right)_{0} \in S$, berlaku:

$$
\left(-r_{1}, r_{1}\right)_{0} \bigodot_{J R-3 C N}\left(-r_{2}, r_{2}\right)_{0} \in S
$$

Selanjutnya, misalkan $\left(-r_{1}, r_{1}\right)_{0} \in S,\left(-r_{2}, r_{2}\right)_{0} \in S$, sehingga diperoleh:

$$
\begin{aligned}
\left(-r_{1}, r_{1}\right)_{0} \bigodot_{J R-3 C N}\left(-r_{2}, r_{2}\right)_{0} & =\left(-r_{1} \cdot-r_{2}+r_{1} \cdot\left(-r_{2}\right)+\left(-r_{2}\right) \cdot r_{1}, r_{1} \cdot r_{2}\right)_{0} \\
& =\left(r_{1} \cdot r_{2}-2\left(r_{1} \cdot r_{2}\right), r_{1} \cdot r_{2}\right)_{0} \\
& =\left(-\left(r_{1} \cdot r_{2}\right), r_{1} \cdot r_{2}\right)_{0}
\end{aligned}
$$

Karena, $-\left(r_{1} \cdot r_{2}\right) \in \mathbb{Z}$ dan $r_{1} \cdot r_{2} \in \mathbb{Z}$. maka dapat diklaim bahwa,

$$
\left(-r_{1}, r_{1}\right)_{0} \bigodot_{J R-3 C N}\left(-r_{2}, r_{2}\right)_{0} \in S .
$$

6.2 Beberapa contoh Ideal dari $\left(\mathrm{JR}-2 \mathrm{CN}, \oplus_{\mathrm{JR}-2 \mathrm{CN}}, \odot_{\mathrm{JR}-2 \mathrm{CN}}\right)$

dan $\left(J R-3 C N, \bigoplus_{J R-3 C N}, \odot_{J R-3 C N}\right)$

Contoh Ideal pada JR - 2CN yaitu :

\section{Preposisi 4}

Subring $\left(K, \bigoplus_{J R-2 C N}, \odot_{J R-2 C N}\right)$ dengan $K=\{(k, k) \in \mathrm{JR}-2 \mathrm{CN}, \forall k \in \mathbb{Z})$

Bukti.

Akan ditunjukkan $K$ adalah Ideal dari JR - 2CN

Misalkan, $(j, r) \in \mathrm{JR}-2 \mathrm{CN}$ dan $(k, k) \in K$. Dimana,

$$
\begin{aligned}
& j=x+\alpha \\
& r=\alpha-x
\end{aligned}
$$

dan

$$
\begin{aligned}
& k=x^{\prime}+\alpha^{\prime} \\
& k=\alpha^{\prime}-x^{\prime}
\end{aligned}
$$

Untuk setiap, $\quad x, x^{\prime}, \alpha, \alpha^{\prime} \in \mathbb{Z}$. Dengan mengeliminasi persamaan (7) dan (8), didapat $x^{\prime}=0$ dan $k=\alpha^{\prime}$. Sehingga,

$$
(j, r) \odot_{J R-2 C N}(k, k)=\left(\frac{j k+r k}{2}, \frac{j k+k r}{2}\right)
$$

Perhatikan,

$$
\begin{aligned}
\left(\frac{j k+r k}{2}\right)^{3}+\left(\frac{j k+k r}{2}\right)^{3} & =\left(\frac{(x+\alpha) \cdot \alpha^{\prime}+(\alpha-x) \cdot \alpha^{\prime}}{2}\right)^{3}+\left(\frac{(x+\alpha) \cdot \alpha^{\prime}+\alpha^{\prime} \cdot(\alpha-x)}{2}\right)^{3} \\
& =\left(\frac{2 \alpha \alpha^{\prime}}{2}\right)^{3}+\left(\frac{2 \alpha \alpha^{\prime}}{2}\right)^{3} \\
& =\left(\alpha \alpha^{\prime}\right)^{3}+\left(\alpha \alpha^{\prime}\right)^{3} \\
& =2\left(\alpha \alpha^{\prime}\right)^{3} \\
& =0+2\left(\alpha \alpha^{\prime}\right)^{3} \\
& =6\left(\alpha \alpha^{\prime}\right)(0)^{2}+2\left(\alpha \alpha^{\prime}\right)^{3}
\end{aligned}
$$


Jelas $\alpha \alpha^{\prime} \in \mathbb{Z}$, sehingga dapat disimpulkan bahwa $\left(\frac{j k+r k}{2}, \frac{j k+k r}{2}\right) \in \mathrm{JR}-2 \mathrm{CN}$. Hal ini juga berarti telah terbukti $(j, r) \odot_{J R-2 C N}(k, k) \subseteq K$, sehingga dapat disimpulkan $K$ merupakan Ideal kiri dari $\mathrm{JR}-2 \mathrm{CN}$.

Selanjutkan, akan ditunjukkan Subring $K$ juga merupakan Ideal kanan dari $J R-2 C N$ atau akan ditunjukkan $K \odot(j, r) \subseteq K$. Misalkan, untuk sebarang $(j, r) \in J R-2 C N$ menggunakan persamaan (5) dan (6) dan untuk sebarang $(k, k)$ menggunakan persamaan (7) dan (8) diperoleh $x^{\prime}=0$ dan $k=\alpha^{\prime}$ sehingga berlaku:

Perhatikan :

$$
\begin{aligned}
(k, k) \bigodot_{\mathrm{JR}-2 \mathrm{CN}}(j, r) & =\left(\frac{k j+k r}{2}, \frac{k r+j k}{2}\right) \\
& =\left(\frac{\alpha^{\prime} \cdot(x+\alpha)+\alpha^{\prime} \cdot(\alpha-x)}{2}, \frac{\alpha^{\prime} \cdot(\alpha-x)+\alpha^{\prime} \cdot(x+\alpha)}{2}\right) \\
& =\left(\frac{2\left(\alpha^{\prime} \alpha\right)}{2}, \frac{2\left(\alpha^{\prime} \alpha\right)}{2}\right) \\
& =\left(\alpha^{\prime} \alpha, \alpha^{\prime} \alpha\right)
\end{aligned}
$$

$$
\begin{aligned}
\left(\frac{k j+k r}{2}\right)^{3}+\left(\frac{k r+j k}{2}\right)^{3} & =\left(\alpha^{\prime} \alpha\right)^{3}+\left(\alpha^{\prime} \alpha\right)^{3} \\
& =0+2\left(\alpha^{\prime} \alpha\right)^{3} \\
& =6\left(\alpha^{\prime} \alpha\right)(0)^{2}+2\left(\alpha^{\prime} \alpha\right)^{3}
\end{aligned}
$$

Karena $\alpha \alpha^{\prime} \in \mathbb{Z}$, maka berlaku $(k, k) \bigodot_{J R-2 C N}(j, r) \subseteq K$, sehingga dapat disimpulkan bahwa $K$ merupakan Ideal kanan.

\section{Preposisi 5}

Subring $\left(M, \oplus_{\mathrm{JR}-2 \mathrm{CN}}, \odot_{\mathrm{JR}-2 \mathrm{CN}}\right)$ dengan $M=\{(m,-m) \in J R-2 C N, \forall m \in \mathbb{Z}\}$

Bukti

Akan ditunjukkan $M$ adalah Ideal pada JR $-2 \mathrm{CN}$. Misalkan $(j, r) \in \mathrm{JR}-2 \mathrm{CN}$ dan $(m,-m) \in M$. dimana,

$$
\begin{aligned}
& j=x+\alpha \\
& r=\alpha-x
\end{aligned}
$$

dan

$$
\begin{aligned}
& m=x^{\prime}+\alpha^{\prime} \\
& -m=\alpha^{\prime}-x^{\prime}
\end{aligned}
$$

Perhatikan bahwa,

$$
(j, r) \odot(m,-m)=\left(\frac{j \cdot m+r \cdot(-m)}{2}, \frac{j \cdot(-m)+m \cdot r}{2}\right)
$$

Selanjutnya, dengan mengeliminasi persamaan (11) dan (12) diperoleh $\alpha^{\prime}=0$ dan $x^{\prime}=m$, sehingga berlaku:

$$
\begin{aligned}
\frac{j \cdot m+r \cdot(-m)}{2} & =\frac{(x+\alpha) \cdot x^{\prime}+(\alpha-x)\left(-x^{\prime}\right)}{2} \\
& =\frac{2\left(x x^{\prime}\right)}{2} \\
& =x x^{\prime} \in \mathbb{Z} \\
\frac{j \cdot(-m)+m \cdot r}{2} & =\frac{(x+\alpha) \cdot\left(-x^{\prime}\right)+x^{\prime} \cdot(\alpha-x)}{2} \\
& =\frac{-2\left(x x^{\prime}\right)}{2} \\
& =-\left(x x^{\prime}\right) \in \mathbb{Z}
\end{aligned}
$$

Jelas $x x^{\prime} \in \mathbb{Z}$, sehingga dapat disimpulkan $(j, r) \odot(m,-m) \subseteq M$ atau dengan kata lain $M$ merupakan Ideal kiri dari JR - 2CN. 
Selanjutnya, akan ditunjukkan Ideal kanan dari JR $-2 \mathrm{CN}$ atau $(m,-m) \odot(j, r) \subseteq M$.

Perhatikan bahwa,

$$
(m,-m) \odot(j, r)=\left(\frac{m \cdot j+(-m) \cdot r}{2}, \frac{m \cdot r+j \cdot(-m)}{2}\right)
$$

Dengan menggunakan persamaan (9) dan (10) untuk $(j, r)$ dan untuk $(m,-m)$ menggunakan persamaan (11) dan (12) diperoleh $\alpha^{\prime}=0$ dan $x^{\prime}=m$. sehingga,

$$
\begin{aligned}
\frac{m \cdot j+(-m) \cdot r}{2} & =\frac{x^{\prime} \cdot(x+\alpha)+\left(-x^{\prime}\right)(\alpha-x)}{2} \\
& =\frac{2 x^{\prime} x}{2} \\
& =x^{\prime} x \in \mathbb{Z} \\
\frac{m \cdot r+j \cdot(-m)}{2} & =\frac{x^{\prime} \cdot(\alpha-x)+(x+\alpha) \cdot\left(-x^{\prime}\right)}{2} \\
& =\frac{-2\left(x^{\prime} x\right)}{2} \\
& =-\left(x^{\prime} x\right) \in \mathbb{Z}
\end{aligned}
$$

Jelas $x, x^{\prime} \in \mathbb{Z}$ sehingga dapat diklaim $(m,-m) \odot(j, r) \subseteq M$ atau dengan kata lain Subring $\mathrm{M}$ merupakan Ideal kanan dari JR - 2CN. Jadi, disini telah dibuktikan bahwa M adalah Ideal dari $\mathrm{JR}-2 \mathrm{CN}$.

Contoh Ideal pada JR - 3CN yaitu,

\section{Preposisi 6}

Subring $\left(S, \oplus_{\mathrm{JR}-3 \mathrm{CN}}, \odot_{\mathrm{JR}-3 \mathrm{CN}}\right)$ dengan $S=\left\{(-s, s)_{0} \in \mathrm{JR}-3 \mathrm{CN}: \forall s \in \mathbb{Z}\right\}$

Bukti

Akan ditunjukkan Subring $S$ adalah Ideal dari JR - 3CN.

Misalkan, $(j, r)_{\alpha} \in R$ dan $(-s, s)_{0} \in S$. dimana, $j=\alpha-r$ dan $s=\alpha^{\prime}-r^{\prime}, s=r^{\prime}$ dan $\alpha^{\prime}=0$. perhatikan bahwa,

$$
\begin{aligned}
(j, r)_{\alpha} \odot(-s, s)_{0} & =(j \cdot(-s)+j \cdot s+(-s) \cdot r, r \cdot s)_{\alpha \cdot 0} \\
& =((-s) \cdot r, r \cdot s)_{0}
\end{aligned}
$$

Sehingga berlaku, $(-s) \cdot r=\left(-r^{\prime}\right) \cdot r=-\left(r^{\prime} \cdot r\right) \in \mathbb{Z}$ dan $r \cdot s=r \cdot r^{\prime} \in \mathbb{Z}$. Karena, $r \cdot r^{\prime} \in \mathbb{Z}$, maka $(j, r)_{\alpha} \odot(-s, s)_{0} \subseteq S$, sehingga jelas $S$ merupakan Ideal kiri dari JR $-3 \mathrm{CN}$.

Selanjutnya, dengan cara yang sama akan dibuktikan Ideal kanan dari JR - 3CN yaitu akan ditunjukkan $(-s, s)_{0} \odot(j, r)_{\alpha} \subseteq S$. Perhatikan,

$$
\begin{aligned}
(-s, s)_{0} \odot(j, r)_{\alpha} & =(-s \cdot j+s \cdot j+r \cdot(-s), s \cdot r)_{0 \cdot \alpha} \\
& =(r \cdot(-s), s \cdot r)_{0}
\end{aligned}
$$

dimana, $r \cdot(-s)=r \cdot\left(-r^{\prime}\right)=-\left(r \cdot r^{\prime}\right) \in \mathbb{Z}$ dan $s \cdot r=r^{\prime} \cdot r \in \mathbb{Z}$.

Sehingga, dapat dikatakan $(-s, s)_{0} \odot(j, r)_{\alpha} \subseteq S$ merupakan Ideal kanan dari JR - 3CN. Jadi, terbukti $\mathrm{S}$ adalah Ideal dari JR - 3CN.

\section{Kesimpulan}

Berdasarkan penelitian serta studi literatur yang telah dilakukan tentang Ring JR - 2CN dan JR - 3CN, dapat ditarik beberapa kesimpulan sebagai berikut :

1. Contoh Subring pada JR $-2 \mathrm{CN}$ dan JR $-3 \mathrm{CN}$ :

- $\quad K=\{(j, j) \in \mathrm{JR}-2 \mathrm{CN}, \forall j \in \mathbb{Z}\}$

- $\quad M=\{(j,-j) \in \mathrm{JR}-2 \mathrm{CN}, \forall j \in \mathbb{Z}\}$

- $\quad S=\left\{(-r, r)_{0} \in \mathrm{JR}-3 \mathrm{CN}, \forall r \in \mathbb{Z}\right\}$ 
2. Contoh Ideal pada JR $-2 \mathrm{CN}$ dan JR $-3 \mathrm{CN}$ :

- $\quad K=\{(k, k) \in \mathrm{JR}-2 \mathrm{CN}, \forall k \in \mathbb{Z}\}$

- $\quad M=\{(m, m) \in \mathrm{JR}-2 \mathrm{CN}, \forall m \in \mathbb{Z}\}$

- $\quad S=\left\{(s, s)_{0} \in \mathrm{JR}-3 \mathrm{CN}, \forall s \in \mathbb{Z}\right\}$

\section{Daftar pustaka}

[1] Alfaris, R., and H. Kamarulhaili. 2011. Two New Abelian Groups Based On JR - 2CN dan JR - 3CN. Aust. J. Basic \& Appl. Sci. 5(11): 2272-2281.

[2] Alfaris, R., and H. Kamarullhaili. 2011. Two New Rings and Field Based On JR - 2CN and JR - 3CN. diseminarkan ICREAM5 ITB; Bandung, 22 Oktober 2011. Hlm 38-39.

[3] Prihandoko, A. 2009. Pengantar Teori Ring dan Implementasinya. Universitas Jember.

[4] Alfaris, R., and H. Kamarulhaili. 2010. Two New Formulas for Generating Infinite Sets of Integer Numbers as a Sum of Two and Three Signed Cubic Numbers. Journal of Mathematics and Statistic. 6(4): 462-467. 